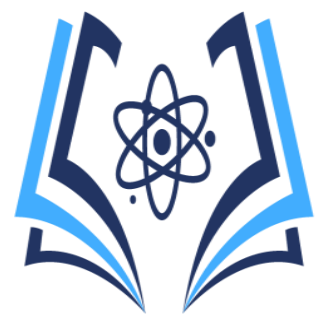

\title{
La obesidad como factor de riesgo de la hipertensión arterial
}

\author{
Obesity as a risk factor for high blood pressure
}

La obesidad como factor de riesgo

\author{
José Jordano Flores Hidalgo(1) \\ María Auxiliadora Guerrero Dueña ${ }^{(2)}$ \\ Reynier García Rodríguez ${ }^{(3)}$ \\ ${ }^{1}$ Instituto Superior Universitario Portoviejo, Manabí, Ecuador. Email: joflogene_2@hotmail.com \\ ${ }^{2}$ Universidad Técnica de Manabí, Portoviejo, Ecuador. Email: joflogene_2@ hotmail.com \\ ${ }^{3}$ Instituto Superior Universitario Portoviejo, Manabí, Ecuador. Email: reygarod79@gmail.com
}

Contacto: reygarod79@gmail.com

\section{Resumen:}

La investigación se basa en la incidencia de la obesidad como factor de riesgo para la hipertensión arterial (HTA) abarcando el análisis de variables de dichos padecimientos, puesto que ambas constituyen factores de riesgo para la salud de las personas. En América Latina, Ecuador es el país que mayor prevalencia posee de HTA debido a que se registra un incremento de motivos de consulta en los centros hospitalarios por HTA en mayor parte en las provincias de Guayas, Pichincha y Manabí y a su vez se estima que 6 de cada 10 adultos poseen sobrepeso u obesidad. Siendo el objetivo del presente trabajo determinar la incidencia de la obesidad como factor de riesgo de la hipertensión en los habitantes de la Ciudadela "Libertad" del cantón Portoviejo en el periodo comprendido de mayo-agosto de 2020. Se realizó un estudio no experimental, inductivodeductivo y transversal a 50 personas. Datos los cuales fueron organizados en una base de datos mediante Microsoft Excel. Finalmente, se obtiene que los datos de la población investigada poseen aspectos positivos y negativos para la salud de las personas. Para el primero se destaca la Frecuencia de Actividad Física y para el segundo los controles médicos y el tratamiento, los cuales representan un porcentaje bajo para lo esperado.

Palabras clave: Hipertensión Arterial, Obesidad, Incidencia, Relación

\section{Abstract:}

The research is based on the incidence of obesity as a risk factor for arterial hypertension (HT), encompassing the analysis of variables in these conditions, since both constitute risk factors for people's health. In Latin America, Ecuador is the country with the highest prevalence of hypertension due to the fact that there is an increase in reasons for consultation in hospital centers for hypertension, mostly in the provinces of Guayas, Pichincha and Manabí, and in turn it is estimated that 6 out of 10 adults are overweight or obese. The objective of this study being to determine the incidence of obesity as a risk factor for hypertension in the inhabitants of the Citadel "Libertad" of the Portoviejo canton in the 
Periodo. Julio - Diciembre 2021

Vol. 5, Nro. 2, Publicado: 2021-12-31

period from July-August 2020. A non-experimental, inductive study was carried out -deductive and transversal to 50 people. Data that were organized in a database using Microsoft Excel, creating tables based on the relationship of the variables. Finally, it is obtained that the data of the investigated population have positive and negative aspects for people's health. For the first, the Frequency of Physical Activity stands out and for the second, the medical controls and treatment, which represent a low percentage for what is expected.

Keywords: Hypertension, Obesity, Incidence, Relationship

\section{Introducción}

La hipertensión y la obesidad constituyen dos problemas de salud pública frecuentes, numerosos estudios realizados en diferentes países coinciden en señalar que existe mayor riesgo en desarrollar hipertensión en pacientes obesos (1). Además, un IMC elevado es un importante factor de riesgo de enfermedades no transmisibles (ENT), como las enfermedades cardiovasculares, que fueron la principal causa de muertes en 2012 junto a la diabetes; los trastornos del aparato locomotor y algunos tipos de cánceres. (2).

Los factores de riesgo en el ámbito metabólico que aumentan la predisposición de desarrollar una ENT son "aumento de la tensión arterial, el sobrepeso y obesidad, la hiperglucemia y la hiperlipidemia. Los cuales, en términos de muertes atribuibles a nivel mundial demuestran que la hipertensión posee el 19\% seguido por el sobrepeso y la obesidad" (3).

Pérez (2012) menciona que, en América Latina, el país con la mayor prevalencia de hipertensión es el Ecuador y la primera causa de muerte es la enfermedad cardiovascular. A su vez, se estima que el $46 \%$ de la población ecuatoriana tiene hipertensión arterial según los estudios hasta el 2012. A su vez, la Encuesta Nacional de Salud y Nutrición (2013) expone que "La prevalencia de hipertensión arterial medida en la población de 18 a 59 años es de $9.3 \%$, siendo de $7.5 \%$ en mujeres y $11.2 \%$ en los hombres." De acuerdo al Registro Diario Automatizado de Consultas y Atenciones Ambulatorias (RDACAA) se reportaron 146.889 atenciones por hipertensión arterial en el 2013;144.457 en el 2014;155.855 en el 2015; 169.437 en el 2016; 217.059 en el 2017 y 63.452 a abril del 2018. Siendo las provincias que registran el mayor número de atenciones por hipertensión: Guayas, Pichincha y Manabí (4).

En 2017 en Manabí se atendieron, 44.341 personas por Hipertensión Arterial según una publicación de El Diario (2018), un diario local de la Provincia. Manabí Noticias, en mayo de 2018 publicó un articulo sobre la temática manifestando el caso de un diagnóstico de una habitante de la provincia expresando que "la manabita tuvo suerte en descubrir a tiempo que padecía esta enfermedad, pues en algunos casos no presenta síntomas y por eso se la conoce como la enfermedad silenciosa."

Mientras que para la prevalencia de obesidad abdominal en la población de 10 a 59 años a escala nacional se obtiene un mayor porcentaje en los últimos decenios. En el quinto decenio de 50 a 59 años $(81.4 \%)$, de 40 a 49 años $(74.6 \%)$ y de 30 a 39 años (67.1\%) (5).

Por otra parte, Machado menciona que, en promedio general, en Ecuador seis de cada 10 adultos sufren de sobrepeso u obesidad. Pues, las tasas de sobrepeso y obesidad en adultos aumentan a un ritmo alarmante y se encuentra en niveles muy altos en las 24 provincias del país [...]. Según sus estadísticas, [...] en provincias como El Oro, Guayas o Imbabura la prevalencia es mayor al 60\% (2019). En lo que respecta a Manabí, como medida de control y prevención la Dirección de Deportes del Gobierno municipal de Manta desarrolla un programa de ejercicios públicos que ayuda a controlar y reducir el sobrepeso de las personas que lo padecen. Se llama 
"Pierda para Ganar" y es aprovechado mensualmente

por unos 300 individuos (6).

Por ende, el presente estudio pretende realizar una investigación de la incidencia que posee la obesidad como factor de riesgo asociado a la hipertensión, quien constituye una de las enfermedades más comunes en conjunto con la Diabetes Mellitus tipo 2 en cuanto a Manabí se refiere. Con la finalidad de describir algunas actividades dirigidas al control y prevención de estos padecimientos.

\section{Materiales y Métodos}

Se realizó un estudio no experimental, inductivodeductivo y transversal a 50 personas adultas, todas habitantes de la Ciudadela "Libertad" del Cantón Portoviejo en la Provincia de Manabí durante el periodo comprendido mayo-agosto de 2020.

Para llevar a cabo, la presente investigación se utiliza la observación y la revisión bibliográfica teórica y estadística para abordar la temática. Pita Fernández \& Pértega Díaz (2001) mencionan que: "los términos estadísticos y epidemiológicos invaden la literatura médica, la medicina es cada vez más cuantitativa, su conocimiento permitirá leer la bibliografía médica con más capacidad crítica para detectar errores potenciales y falacias."

El tratamiento estadístico se llevará a cabo utilizándose como instrumento de recolección de datos para complementar la información investigada, la aplicación de encuestas. Teniéndose como variables: Género, Edad, Hipertensión Arterial, Índice de Masa Corporal (IMC), Frecuencia de Actividad Física, Prioridad de alimentos y algunos conocimientos en base al tópico, siendo la mayoría de carácter cualitativa, por ende, se aplicó estadística descriptiva para su análisis.

Los datos, fueron organizados en una base de datos a través de Microsoft Excel y posteriormente se filtraron los mismos permitiendo crear tablas de frecuencia en base a la relación de variables para la obtención de frecuencias absolutas y porcentualesque permitieron realizar un análisis que será expuesto en los siguientes puntos.

\section{Resultados}

En el periodo comprendido entre mayo-agosto de 2020 se logró la concreción de la investigación a 50 individuos de una comunidad. Los cuales se encuentran en una media que rodea los 52,8 años de edad, teniéndose en el gráfico 1 como mínimo 37 años y como máximo 72 , de entre los cuales la sumatoria del género masculino corresponde al 56\% y el $44 \%$ restante de la población total pertenece al género femenino.

El 74\% de los habitantes padecen de Hipertensión Arterial (HTA) desglosando el tiempo en que fueron diagnosticados con la enfermedad. A esto se suma que el $44 \%$ de los mismos presentan índices de sobrepeso y obesidad, presentándose en detalle la Tabla 2. Esto debido a que los datos del IMC de la población oscilan a partir de un mínimo de $19 \mathrm{~kg} / \mathrm{m}^{2}$ lo cual se considera en un peso normal (Normopeso) y un máximo de $37 \mathrm{~kg} / \mathrm{m}^{2}$ lo cual se clasifica en Obesidad Tipo 2. Obteniéndose a su vez una media poblacional para el IMC de $25,9 \mathrm{~kg} / \mathrm{m}^{2}$. El 97\% realizan durante la semana alguna actividad física. Destacando la frecuencia de ejercicio de 2 a 3 veces por semana con un $46 \%$ del total de la población.

El $82 \%$ de la población en estudio que corresponde a una frecuencia de 41 personas, poseen antecedentes de sobrepeso y/u obesidad familiar. Añadiendo que un $66 \%$ del total poblacional no asistió a controles médicos durante un intervalo de 3 meses. En cuanto a si recibieron algún tratamiento durante el mismo intervalo de tiempo el $46 \%$ manifestó no administrarse alguno.

Al interrogar si los individuos consideran consumir una dieta balanceada como medida preventiva para la obesidad se obtuvo que el $98 \%$ de la población 
considera que, sí. A su vez, se manifiesta que el $72 \%$ de la población prefiere no consumir comida chatarra o grasosa durante la semana. Datos de los cuales el $29 \%$ de los individuos consumen dichas comidas y padecen de HTA.

En relación a la alimentación, se les pidió a los individuos que ordenaran del 1 al 6 los grupos de alimentos que se les presentó, considerando un orden ascendente, donde 1 sea lo que más consume y 6 , lo menos. Obteniéndose 2 tablas donde, la primera mostraba los datos que proporcionaron los pacientes y la segunda el orden de prioridad de grupos de alimentos.

Finalmente se añade que el $82 \%$ de la población posee el conocimiento de que la obesidad es un factor de riesgo para la HTA y a su vez, el $92 \%$ conoce las consecuencias de la obesidad

\section{Discusión}

La obesidad y la hipertensión constituyen factores de riesgos predominantes en la salud. Según (1) "La relación entre obesidad e HTA tiene una especial relevancia por su magnitud y por las repercusiones que tiene en la clínica diaria". La presente investigación revela que el $22 \%$ de la población hipertensa posee sobrepeso y otro $22 \%$ poseen algún tipo de obesidad. De Luis Román, Aller, \& Bustamante (2008) en parte concuerdan con los resultados de la investigación al manifestar que "un elevado porcentaje de pacientes hipertensos son obesos" y por ello exponen que la reducción de peso con dietas hipocalóricas se ha demostrado como una opción terapéutica.

Probablemente el abordaje dietético más complejo se ha realizado mediante la dieta DASH (Dietary Approaches to Stop Hypertension). Esta aproximación se caracteriza por una dieta rica en frutas, vegetales y lácteos descremados, que incluye cereales, pollo, pescado y nueces, y que contiene pequeñas cantidades de carnes rojas, dulces y bebidas azucaradas, disminuyendo sustancialmente la presión
Vol. 5, Nro. 2, Publicado: 2021-12-31

arterial en personas hipertensas y aquéllas sin hipertensión (7).

Entre los tipos de alimentos, la muestra permitió identificar un mayor consumo de Carbohidratos denominándose así a las harinas refinadas, verde y arroz. Un porcentaje elevado (88\%) en relación a las frutas $(62 \%)$ y verduras $(58 \%)$, esto en gran parte podría deberse a la situación socioeconómica que afronta el país y a su vez se añade que la dieta y gastronomía manabita se basa sobre todo en las características antes mencionadas debido a la estrecha conexión que posee con la historia y tradición por ello se cita a (8) quien en su libro "Manabí y su comida milenaria" manifiesta que "Manabí es una provincia que guarda como un tesoro la sabiduría de toda una tradición culinaria, una tradición que se remonta a miles de años, de la cual son herederos en la actualidad los y las manabitas, y de la que constantemente hacen gala ante sus convidados." A su vez menciona que la comida posee relación con la alianza social, la religión, la muerte, el amor, la pasión y la jerarquía social. Utilizando como ingredientes básicos el maíz, maní, cacao, zapallo, yuca, frijoles, plátano, camote, jíquima y sidrilla.

El plátano verde es uno de los alimentos más utilizados en la cocina manabita, de hecho ha tomado un gran papel ante la pandemia Covid-19. Mencionando Zapata Mora (2020) que: “el plátano, desde que arrancó la cuarentena, se ha convertido en un producto apetecido sobre todo en la costa ecuatoriana, al punto de ser intercambiado con pescado". Según el estudio "Cuantificación de almidón total y de almidón resistente en harina de plátano verde (Musa cavendishii) y banana verde (Musa paradisíaca)" se llega a la conclusión de que tanto el plátano como la banana verde representan una buena fuente de almidón resistente que pueden ser aprovechados en la preparación de diversos productos propios de la región. Además, las 
Periodo. Julio - Diciembre 2021

investigaciones reportan que es muy beneficioso por Vol. 5, Nro. 2, Publicado: 2021-12-31 sus efectos fisiológicos en el organismo como disminución del tiempo de tránsito intestinal, reducción de glucosa en sangre y consecuentemente, la cantidad del nivel de colesterol (9).

En gran parte, se demuestra que, los habitantes de la ciudadela Libertad realizan actividad física bien sea 1 vez, 2 o 3 veces e incluso 4 o más durante la semana. Lo que expone un aspecto positivo para la salud de los habitantes. De Luis Román, Aller, \& Bustamante (2008) mencionan que: "Los estudios de intervención han demostrado que la realización de actividad física produce un descenso en la tensión arterial sistólica y diastólica de aproximadamente 6$8 \mathrm{mmHg}$. Los sujetos sedentarios tienen hasta casi un 50\% de posibilidades de desarrollar HTA".

Algo importante para acotar es la percepción que posee la muestra expresando en gran porcentaje que una dieta balanceada es una excelente medida preventiva para la hipertensión y la obesidad. No obstante, existe un porcentaje, mínimo (29\%), que posee hipertensión y menciona consumir comidas chatarras y grasosas durante la semana. Siendo una limitación para evaluar y analizar dichas variables, añadiendo los sesgos de error que se presenta debido a la metodología utilizada, puesto que, las encuestas presentan errores en sus resultados debido a la posible falsedad de las respuestas por la deseabilidad social. Por ende, las investigaciones posteriores deben ahondar un poco más en el asunto para resolver la hipótesis "El consumo de comidas hipercalóricas es frecuente en la vida cotidiana de pacientes hipertensos que poseen un IMC elevado".

La reducción del consumo de grasa saturada se asocia a un descenso en los niveles tensionales, mientras que el mayor consumo de ácidos grasos poliinsaturados se ha relacionado con el mismo efecto favorable. Acotando que entre las grasas poliinsaturadas se encuentran (grasas de semillas-omega 6 y de pescados-omega 3) (7).

Freire, y otros (2013) consideran a la obesidad central medida mediante la circunferencia de la cintura y al menos dos de los siguientes componentes: triglicéridos superiores a $150 \mathrm{mg} / \mathrm{dl}$, lipoproteínas de alta densidad (HDL) inferior a $40 \mathrm{mg} / \mathrm{dl}$ para hombres y $50 \mathrm{mg} / \mathrm{dl}$ para mujeres, hipertensión superior a 130/80 $\mathrm{mmHg}$ y glucosa en plasma superior a $100 \mathrm{mg} / \mathrm{dl}$. Lo cual representa una limitación evidente para la investigación, debido a que no se disponen de los recursos económicos para realizar estudios de laboratorio y a esto se suma la presente pandemia del COVID-19, donde lo ideal es mantener un distanciamiento social para evitar un posible contagio para evitar el incremento de casos positivos.

Los resultados exponen que un total de 23 personas equivalentes al $46 \%$ no han tomado medicaciones durante 3 meses añadiendo que el $68 \%$ de la población no ha asistido a controles médicos en el mismo intervalo de tiempo. Entonces para ello se plantean hipótesis a manera de interrogación: ¿Será la pandemia del COVID-19 un factor coadyuvante para que las personas no asistan a una consulta médica? O ¿Serán los recursos socioeconómicos quienes estarán más asociados a este fenómeno y utilizarán otro tipo de medicina alternativa para tratarse?

Esto se plantea puesto que, en 2018 Herrera, Rodríguez, \& Torregrosa. Realizaron un estudio donde exponen que "El $58 \%$ de la población entrevistada, teniendo una muestra de 614 individuos, utiliza plantas medicinales para tratar la hipertensión, siendo las más utilizadas Matricaria recutita (manzanilla), Plectranthus amboinicus (oreganón), Cymbopogon citratus (hierba luisa) y Valeriana officinalis (valeriana)" Manifestando a su vez que la hierba luisa es la única con eficacia comprobada como anti-hipertensiva. En donde los autores mencionados concluyen que "El uso popular de tres de las plantas estudiadas se atribuye a sus 
Periodo. Julio - Diciembre 2021

Vol. 5, Nro. 2, Publicado: 2021-12-31

efectos sedante y relajante, por lo que sus efectos antihipertensivos son un mito y no una realidad".

Pardell H y otros (1968, citado en De Luis Román, Aller, \& Bustamante, 2008) mencionan que: Ante un panorama de dimensiones pandémicas, los tratamientos farmacológicos suponen grandes costes directos para los Sistemas Públicos de Salud, por ello las medidas no farmacológicas son cada vez más importantes en el tratamiento integral de estos pacientes, incluyendo la reducción de peso, el ejercicio físico, la disminución de ingesta de alcohol, tabaco y café, aumento de consumo de calcio, magnesio, potasio y fibra, así como las dietas pobres en grasas y la restricción de sal.

López de Fez, Gaztelu, Rubio, \& Castaño, (2004) mencionan que: es fácil asumir, que una corrección del sobrepeso, será beneficioso en la reducción de cifras de tensión arterial. Para ello existen medidas no farmacológicas y farmacológicas. Las primeras consisten en una dieta baja en grasa, pobre en sal, moderada en hidratos de carbono y rica en fibra; ejercicio aeróbico y disminución de ingesta de alcohol, entre otras. Y las farmacológicas incluyen la introducción de los inhibidores de la enzima de conversión de la angiotensina (IECA) para la hipertensión y si se añade la obesidad se incluyen los diuréticos, betabloqueantes, alfabloqueantes y calcioantagonistas.

Para la búsqueda de soluciones a los problemas de sobrepeso y obesidad en el Ecuador es necesaria la presencia de cuatro actores según la Revista Informativa de la OPS/OMS Representación Ecuador (2014):

Primero. El Estado con su responsabilidad reguladora y de control sobre el etiquetado de alimentos y la propaganda engañosa de alimentos no saludables. Pero además, con la responsabilidad de proteger la salud de la población, debe promover el consumo de agua, y la eliminación de bebidas endulzadas (jugos artificiales y gaseosas), el consumo de la comida ecuatoriana saludable (sopas como repe, ají de carne, sancocho, arroz de cebada, viche; pescado, carnes rojas magras) preparando los platos típicos pero sustentados en buenas prácticas nutricionales, evitando las "hiperporciones"; el consumo de cereales complejos como quínoa, chochos, amaranto, leguminosas como fréjol, garbanzo, lenteja en la alimentación diaria; el consumo de aceites poli y monoinsaturados: aceite de maíz, soya, canola, oliva y, un menor consumo de alimentos hiperprocesados y bebidas y jugos artificiales que tienen alta concentración de azúcar. Para priorizar estos cambios en los hábitos alimentarios es necesario tomar ciertas medidas:

- Aumentar la disponibilidad y accesibilidad al agua potable.

- Imponer un impuesto a las bebidas endulzadas, gaseosas y jugos artificiales.

- Regular el contenido de azúcar, sal y grasa añadidas.

- Proponer y promover la disminución del tamaño de las porciones en restaurantes y lugares de expendio de comida preparada e industrializada.

- Reducir el costo de los aceites saludables: aceite de maíz, soya, canola, oliva.

- Limitar la cantidad de sodio adicionada a los alimentos y reducir su consumo.

- Aumentar la oferta de leche descremada y derivados.

Segundo. El sector privado debe ajustarse a las normas y regulaciones, “adoptando prácticas responsables", particularmente respecto a la promoción y la comercialización de alimentos con alto contenido de grasas saturadas, ácidos grasos trans, azúcares libres, sal. Este actor debe evitar generar conflictos de interés entre los profesionales de la salud y la industria (10).

Tercero. La sociedad civil organizada y empoderada de sus derechos, vigilante permanente de la 
Periodo. Julio - Diciembre 2021

Vol. 5, Nro. 2, Publicado: 2021-12-31

aplicación de normas, requerimientos y reglamentos, con acceso a información basada en la evidencia científica; que exija información no sesgada, que controle la propaganda engañosa de productos alimentarios (10).

Cuarto. Los profesionales de la salud, y en particular los médicos, deben estar capacitados para hacer consejería nutricional e incluirla entre sus actividades profesionales, deben proveer información sustentada en la evidencia científica, y promover comportamientos saludables en la población (10).

En cuanto a las acciones que promueve el Ministerio de Salud Pública (s.f.) para la Hipertensión en el Ecuador se mencionan:

- Evitar el consumo de cigarrillo y bebidas alcohólicas.

- Mantener el peso corporal dentro de los límites normales.

- Consumir un régimen rico en frutas, verduras, fibras, lácteos con bajos porcentajes de grasas.

- Disminución del consumo de sal a 5gr/día ha demostrado permitir una reducción hasta en un $10 \mathrm{mmHg}$ de presión arterial.

- Jornada de ejercicio físico para evitar el sedentarismo, mantener el cuerpo sano y libre de estrés.
La población estudiada posee aspectos positivos y negativos en relación a la temática abordada, muchos de estos aspectos pueden estar influenciados por las medidas preventivas aplicadas por la pandemia Covid-19. Entre los aspectos negativos se encontró que los individuos presentan índices de masa corporal elevados, añadiendo que una gran parte posee hipertensión arterial y no han asistido a consulta médica y mucho menos han tomado un tratamiento específico para sus enfermedades, lo cual representa un riesgo para la salud de las personas.

Entre los aspectos positivos, se revelan altos índices de frecuencia de actividad física que podrían complementarse perfectamente con una dieta balanceada de acuerdo a la percepción de los habitantes. No obstante, la dieta es uno de los aspectos más complicados de manejar debido al sesgo de error que presenta debido a la falsedad de las respuestas por la deseabilidad social, todas estas se encuentran asociadas a un nivel socioeconómico medio o bajo, puesto que es importante reconocer que una dieta balanceada requiere de la inversión de algo de dinero y tiempo para la preparación. Concluyendo finalmente que, existe incidencia de la obesidad ante la HTA y a su vez, ambos padecimientos presentan factores modificables que deben seguirse promocionando en los habitantes.

\section{Conclusiones}

\section{Referencias}

1. Bastidas R, Castaño J, Enríquez D, Giraldo J, González J, Güependo D, et al. Relación entre hipertensión arterial y obesidad en pacientes hipertensos atendidos en ASSBASALUD ESE, Manizales (Colombia) 2010. Archivos de Medicina. 2011 julio-diciembre; 11(2): p. 150-158.

2. OMS. Organización Mundial de la Salud. [Online].; 2020. Available from: https://www.who.int/es/newsroom/fact-sheets/detail/obesity-and-overweight.

3. OMS. Organización Mundial de la Salud. [Online].; 2018. Available from: https://www.who.int/es/newsroom/fact-sheets/detail/noncommunicable-diseases.

4. Ministerio de Salud Pública. Coordinación Zonal 1 - Salud. [Online].; 2019. Available from: http://www.saludzona1.gob.ec/cz1/index.php/sala-de-prensa/846-prevencion-y-control-de-hipertensionarterial-llega-a-barrios-rurales-de-esmeraldas.

5. Freire W, Ramírez M, Belmont P, Mendieta M, Silva K, Romero N, et al. ENSANUT: Encuesta Nacional de Salud y Nutrición. [Online].; 2013. Available from: https://www.ecuadorencifras.gob.ec/documentos/webinec/Estadisticas_Sociales/ENSANUT/Publicacion\%20ENSANUT\%202011-2013\%20tomo\%201.pdf. 
6. Risco Intriago. J. Desafío a las personas con sobrepeso: “Pierda para Ganar”. Revista de Manabí. 2019 Enero 28.

7. de Luis Román D, Aller R, Bustamante J. Aspectos terapéuticos de la dieta en la hipertensión arterial. NefroPlus. 2008; 1(1): p. 1-55.

8. Regalado Espinoza L. Manabí y su comida milenaria. Segunda ed. Manta: Universidad Laica Eloy Alfaro de Manabí; 2019.

9. Soto Azurduy V. Cuantificación de almidón total y de almidón resistente en harina de plátano verde (Musa cavendishii) y banana verde (Musa paradisíaca). Revista Boliviana de Química. 2010 diciembre 05; 27(2): p. 94-99.

10. Representación OPS/OMS - Ecuador. Revista Informativa OPS/OMS: Representación Ecuador. Revista Informativa OPS/OMS. 2014 Junio;(32): p. 13. 